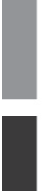

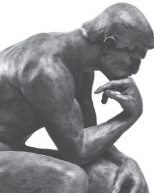 \\ THE SAGE PROGRAM \\ Survey of Access to GastroEnterology}

\section{The Survey of Access to GastroEnterology (SAGE) Program: Advocacy needs facts not anecdotes}

Desmond Leddin MB FRCPC MRCPI MSC

For the SAGE Advisory Group: Jan Irvine, Des Leddin, Victor Plourde, Craig Render, Carlo Fallone

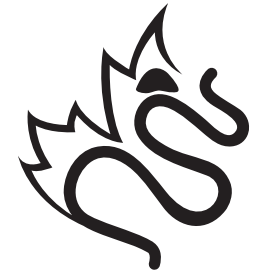

Canadian Association of Gastroenterology

En français voir page 942

In partnership with provincial gastroenterology societies, the Canadian Association of Gastroenterology (CAG) plans to update the national dataset of wait times for access to digestive disease consultation and endoscopy.

The new program is called the Survey of Access to GastroEnterology (SAGE).

A sage is a person of gravity and wisdom. This seems appropriate because results from the 2005 survey of wait times were certainly grave and, undoubtedly, wisdom is needed to improve health care access.

The 2005 Practice Audit in Gastroenterology (PAGE) Wait Times program (1) showed that only a minority of patients was seen within the target or benchmark times established by a consensus group (2). Those data allowed the CAG to lobby nationally, and the provincial societies to lobby regionally, for the resources needed to correct the problem.

The PAGE data are now three years old and an updated view of wait times is essential. Data are needed to advocate for patients by presenting facts, not anecdotes, to the health care administration at both a government and hospital level.

Is this situation still grave? We do not know for

\section{What is SAGE?}

SAGE is an initiative of the CAG and provincial gastroenterology societies, which consists of a short survey that will provide a snapshot of how long Canadians wait for digestive and liver disease consultation and procedures.

\section{What is involved?}

Specialists will record four to five data points on at least five new consecutive clinic patients (consults) and five new consecutive procedure patients (less than 1 minute per patient entry).

\section{Who should participate?}

Gastroenterologists and internists specializing in gastroenterology, provided they record the date that patients are first referred.

\section{When is SAGE?}

SAGE week is November 17 to 21 ; the alternate week for SAGE completion is November 24 to 28.

\section{Where do I get more details?}

Visit the CAG Web site to read more about SAGE and try the demo online survey. You may also print an information package on SAGE to share with colleagues at:

\section{www.cag-acg.org, and click on the SAGE logo}

\section{How do I sign up?}

Contact the CAG National Office to specify whether you prefer to complete the survey online (preferred) or on paper. You will be e-mailed the URL to the final survey or the paper form (as preferred) before November 17 .

E-mail: SAGE@cag-acg.org

Toll-free: 1-888-780-0007

Fax: 905-829-0242

The CAG is proud to acknowledge its Benefactor Corporate Sponsors:

Abbott Canada
Pentax Canada Inc

\begin{abstract}
AstraZeneca Canada Inc
AstraZeneca Canada Inc
Procter \& Gamble Pharmaceuticals
\end{abstract}

Olympus Canada Inc Schering-Plough Canada Inc UCB Pharma Inc 


\section{Le programme d'Enquête sur l'accès à la} gastroentérologie (EAGE) : En défense d'intérêts, il

\section{faut des faits, pas des données empiriques}

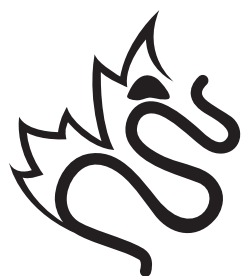

L'Association Canadienne de Gastroentérologie

For English see page 941

Desmond Leddin MB FRCPC MRCPI M. Sc.

Pour le groupe consultatif de I'EAGE : Jan Irvine, Des Leddin, Victor Plourde, Craig Render, Carlo Fallone

Gn partenariat avec les sociétés provinciales de gastroentéroloEgie, l'Association canadienne de gastroentérologie (ACG) prévoit mettre à jour l'ensemble de ses données nationales sur les temps d'attente pour accéder à des consultations et à des endoscopies pour les maladies digestives.

Le nouveau programme se nomme Enquête sur l'Accès à la GastroEntérologie (EAGE).

En anglais, l'acronyme de ce programme est SAGE, un être grave qui détient la sagesse. Ça tombe bien, car les résultats de l'enquête sur les temps d'attente de 2005 étaient bel et bien graves, et de toute évidence, il faut de la sagesse pour améliorer l'accès aux soins.

Les temps d'attente dans le programme de vérification de la pratique en gastroentérologie (PAGE) de 2005 (1) a révélé que seule une minorité de patients voyaient le médecin dans les délais ciblés ou normaux établis par un groupe consensuel (2). Ces données ont permis à l'ACG d'exercer des pressions sur la scène nationale, et aux sociétés provinciales d'en exercer dans les régions, afin d'obtenir les ressources pour corriger le problème.

Les données de PAGE ont maintenant trois ans, et une

\section{Qu'est-ce que l'EAGE ?}

L'EAGE est une courte enquête qui fournit un aperçu du temps d'attente des Canadiens avant d'obtenir une consultation ou de subir une intervention dans le domaine des maladies digestives et hépatiques.

\section{Ce qu'elle exige ?}

Les spécialistes répertorieront de quatre à cinq points de données sur au moins cinq nouveaux patients cliniques consécutifs (consultations) et cinq nouveaux patients consécutifs qui subissent une intervention (moins d'une minute par saisie de données sur un patient).

\section{Qui devrait participer?}

Les gastroentérologues et les internistes spécialisés en gastroentérologie, pourvu qu'ils précisent la date à laquelle leurs patients ont été aiguillés pour la première fois.

\section{Quand a lieu l'EAGE ?}

La semaine de l'EAGE a lieu du 17 au 21 novembre. Une deuxième semaine pour remplir l'EAGE est prévue du 24 au 28 novembre.

\section{Où puis-je obtenir plus d'information ?}

Consultez le site Web de l'ACG pour en savoir plus au sujet de l'EAGE et essayer le modèle d'enquête virtuelle, offert en français. Vous pouvez également imprimer une trousse d'information en anglais au sujet de l'EAGE, à l'adresse www.cag-acg.org, et cliquer sur le logo de l'EAGE.

\section{Comment puis-je m'inscrire ?}

Communiquez avec le bureau national de l'ACG pour préciser si vous préférez remplir l'enquête virtuelle (préconisée) ou la copie papier. Vous recevrez par courriel l'adresse Internet ou la copie papier de l'enquête définitive, à votre choix, avant le 17 novembre.

$$
\begin{aligned}
& \text { Courriel : SAGE@cag-acg.org } \\
& \text { Sans frais : 1-888-780-0007 } \\
& \text { Télécopieur : 905-829-0242 }
\end{aligned}
$$

s'impose. Pour défendre les administration de la santé du gouvernement et des hôpitaux, et non des données empiriques.

La situation est-elle toujours grave? Nous n'en sommes pas certains, mais nous savons que la situation canadienne des effectifs médicaux continue d'être inquiétante. D'après l'Institut canadien d'information sur la santé, le Canada possède le moins de médecins pour cent mille habitants que tout autre pays industrialisé, à l'exception du Japon (3). De plus, la population vieillissante des baby-boomers a une incidence sur les consultations en gastroentérologie qui, on le sait, augmentent proportionnellement à l'âge des patients. Nous pouvons donc prévoir que la demande de services en gastroentérologie augmentera, alors même que les effectifs médicaux sont limités, ce qui remet en question notre capacité de répondre aux besoins des patients.

Nous invitons tous les gastroentérologues cliniques et les internistes qui voient des patients en gastroentérologie à participer à l'EAGE.

suite à la page 943

L'ACG est fière de présenter ses bienfaiteurs :
Abbott Canada
AstraZeneca Canada Inc
Axcan Pharma Inc
Olympus Canada Inc
Pentax Canada Inc
Procter \& Gamble Pharmaceuticals
Schering-Plough Canada Inc 


\section{Continued from page 941}

If a large number of clinicians participate, we will have an extremely powerful dataset. Regional results can be provided to provincial gastroenterology societies to enable them to advocate for resources in their region. National findings will form the basis of the CAG's efforts to continue to lobby the federal government to increase the profile of gastroenterology and resources for patients.

We hope that you will plan to take part in SAGE this November. Your data are crucial and will ultimately allow us to advocate for better care for your patients.

\section{REFERENCES}

1. Leddin D, Armstrong D, Barkun AN, et al. Access to specialist gastroenterology care in Canada: Comparison of wait times and consensus targets. Can J Gastroenterol 2008;22:161-7.

2. Paterson WG, Depew WT, Paré P, et al. Canadian consensus on medically acceptable wait times for digestive health care. Can J Gastroenterol 2006;20:411-23.

3. Canadian Institute for Health Information. Canada's Health Care Providers 2007. Full Report December 3, 2007.

$<$ http://secure.cihi.ca/cihiweb/dispPage.jsp?cw_page=PG_874_E\&cw_ topic $=874 \&$ cw_rel=AR_35_E $>$ (Version current at August 12,2008$)$. suite de la page 942

L'enquête elle-même est très directe. Les cliniciens seront invités à fournir des données sur cinq nouveaux patients cliniques et cinq nouveaux patients subissant une endoscopie ou une autre intervention. Nous demandons seulement la tranche d'âge du patient, la date et la raison de l'aiguillage et la date de l'intervention ou de la consultation. Aucune donnée nominative n'est colligée au sujet du patient, et l'EAGE peut être remplie par Internet ou sur papier. C'est aussi simple que ça.

Si un grand nombre de cliniciens participent, nous disposerons d'un ensemble de données extrêmement puissant. Les résultats régionaux pourront être fournis aux sociétés provinciales de gastroentérologie afin de leur permettre de prôner l'attribution de ressources dans leur région. Les résultats nationaux orienteront l'exercice des pressions de l'ACG auprès du gouvernement fédéral afin qu'il accroisse le profil de la gastroentérologie et les ressources pour les patients.

Nous espérons que vous planifierez de participer à l'EAGE en novembre. Vos données sont essentielles et en définitive, elles nous permettront de préconiser de meilleurs soins pour vos patients.

\section{RÉFÉRENCES}

1. Leddin D, Armstrong D, Barkun AN et coll. Access to specialist gastroenterology care in Canada: Comparison of wait times and consensus targets. Can J Gastroenterol 2008;22:161-7.

2. Paterson WG, Depew WT, Paré P et coll. Canadian consensus on medically acceptable wait times for digestive health care. Can J Gastroenterol 2006;20:411-23.

3. Institut canadien d'information sur la santé. Les dispensateurs de soins au Canada 2007. Rapport en entier, le 3 décembre 2007. $<$ http://secure.cihi.ca/cihiweb/dispPage.jsp?cw_page=PG_874_F\&cw_ topic $=874 \& c w \_r e l=A R \_35 \_F>$ (version à jour le 12 août 2008). 


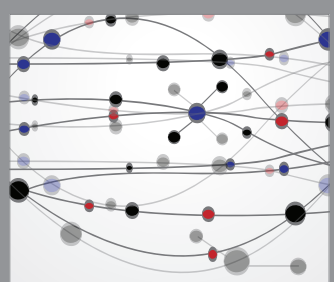

The Scientific World Journal
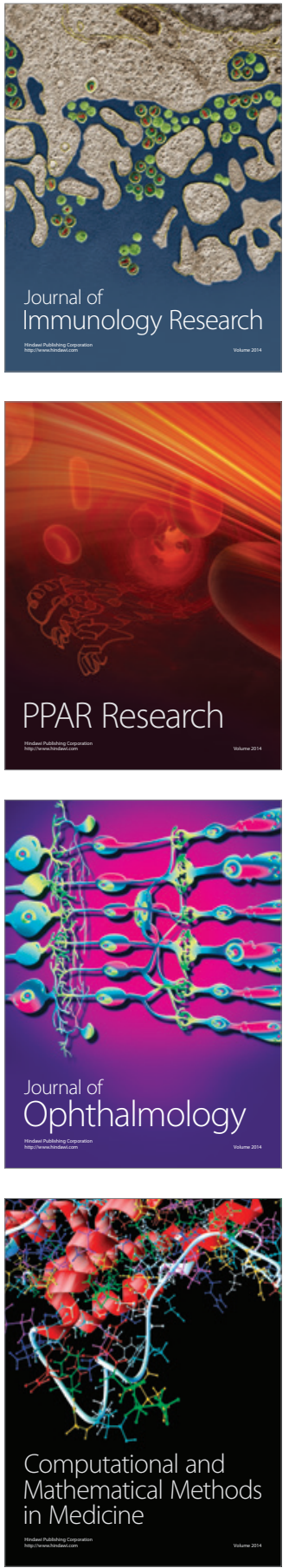

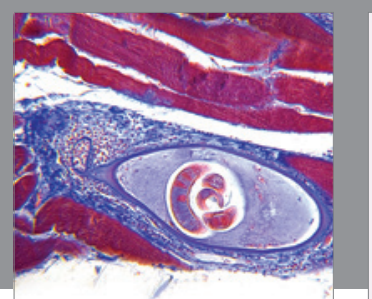

Gastroenterology Research and Practice

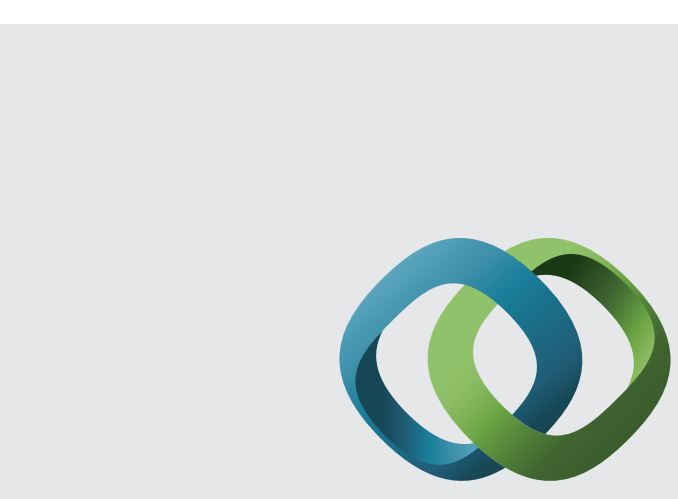

\section{Hindawi}

Submit your manuscripts at

http://www.hindawi.com
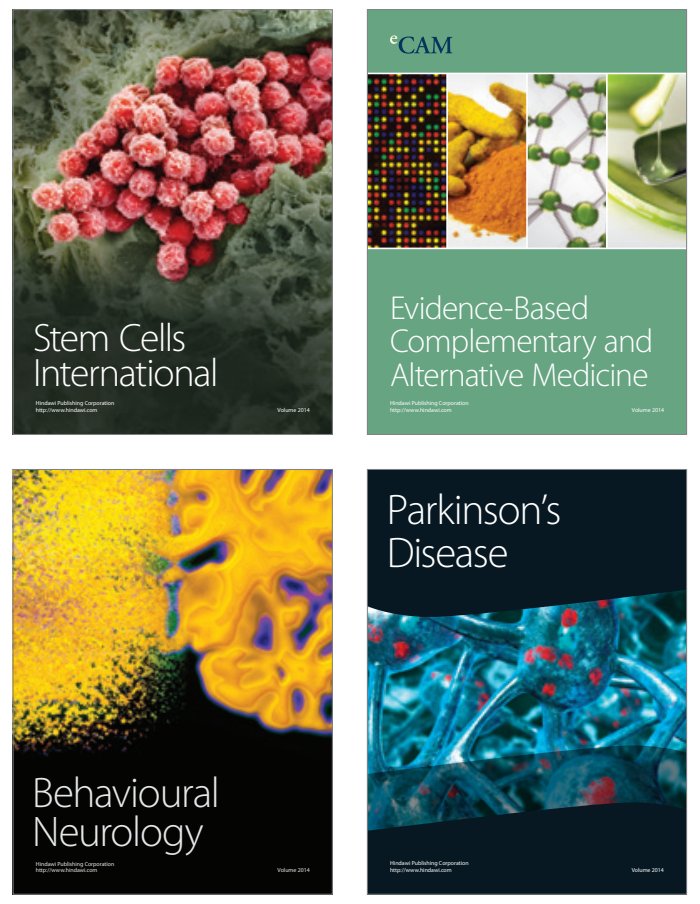
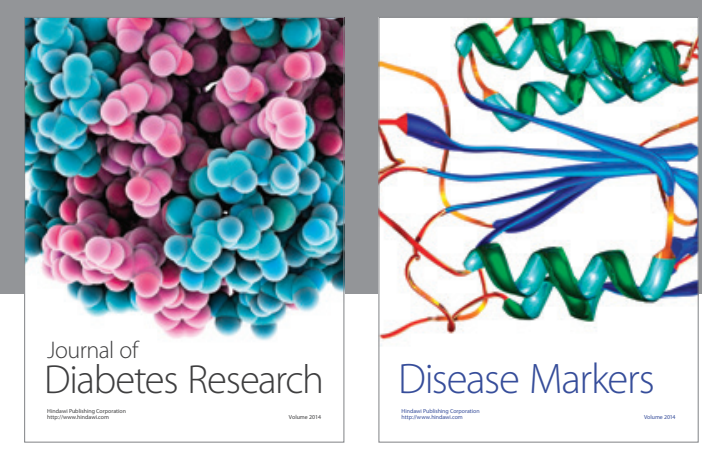

Disease Markers
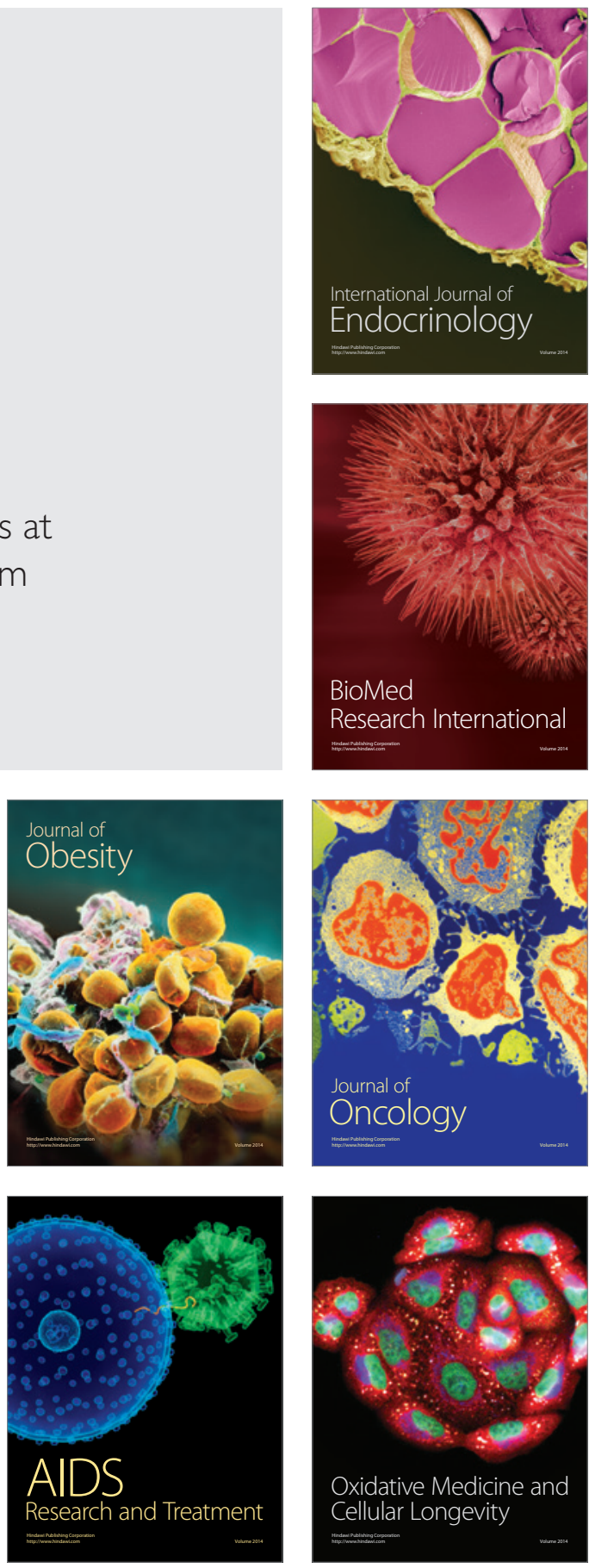